\title{
Relating microwave backscatter azimuth modulation to surface properties of the Greenland ice sheet
}

\author{
Ivan S. ASHCRAFT, David G. LONG \\ Microwave Earth Remote Sensing Laboratory, Brigham Young University, 459 Clyde Building, Provo, Utah 84602, USA \\ E-mail: long@ee.byu.edu
}

\begin{abstract}
Azimuth dependence of a normalized radar cross-section $\left(\sigma^{\circ}\right)$ over the Greenland ice sheet is modeled with a simple surface scattering model. The model assumes that azimuth anisotropy in surface roughness at scales of $\mathbf{3 - 3 0 0 ~} \mathrm{m}$ is the primary mechanism driving the modulation. To evaluate the contribution of azimuth anisotropy in surface roughness to the radar backscatter, the model is compared to models based on isotropic surface roughness. The models are inverted to estimate snow surface properties using $\sigma^{\circ}$ measurements from the C-band European Remote-sensing Satellite advanced microwave instrument in scatterometer mode. Results indicate that the largest mesoscale rms surface slopes are found in the lower portions of the dry snow zone. Estimates of the preferential direction in surface roughness are highly correlated with katabatic wind fields over Greenland, which is consistent with wind-formed sastrugi as the dominant mechanism causing azimuth modulation of $\sigma^{\circ}$. The maximum improvement of the azimuth modulation surface model compared to its isotropic counterparts occurs in the lower regions of the dry snow zone where the azimuth variability of $\sigma^{\circ}$ is the largest. In regions with azimuth modulation over $1 \mathrm{~dB}$, the mean root-mean-square error estimate of the azimuth-dependent surface scattering model is $0.46 \mathrm{~dB}$ compared with $0.70 \mathrm{~dB}$ for similar models using isotropic roughness.
\end{abstract}

\section{INTRODUCTION}

The Greenland ice sheet is a critical area of study for estimating effects of global climate change. Remote sensing is an essential tool for studying the dynamics of this region, as only a limited number of in situ measurements are available due to the considerable effort associated with on-site studies. In many ways, satellite-based measurements of the normalized radar cross-section $\left(\sigma^{\circ}\right)$ are optimal for monitoring this region. Scatterometers provide complete coverage every few days or, with some sensors, multiple times per day. Quality scatterometer measurements are obtained in most weather conditions, day and night. The $\sigma^{\circ}$ values are sensitive to snow grain-size, snow wetness and subsurface features, making it possible to map the Greenland ice facies (Long and Drinkwater, 1994), estimate accumulation (Drinkwater and others, 2001) and measure melt extent and duration (Wismann, 2000; Ashcraft and Long, in press).

Understanding the relationship between $\sigma^{\circ}$ azimuth dependence and physical properties of the surface can be used to better understand the dynamics of the ice sheet. Past studies employing $\sigma^{\circ}$ measurements over Greenland have assumed azimuth modulation to be negligible. However, as remote-sensing studies become more refined, extracting the relationship between the azimuth variation and surface features is necessary for characterizing processes related to long-term change. Our investigations have found azimuth modulation to be relatively stable over annual cycles, making modulation changes good indicators of long-term change. The key lies in relating this change to surface properties.

This paper presents a simple surface scattering model relating physical properties of the surface to observed azimuth modulation of $\sigma^{\circ}$. We first present a background discussion of the data, the properties of the Greenland ice sheet and previous research on azimuth modulation. Next, a simple surface scattering model is described which includes azimuth modulation. This model is inverted using data from two study locations and the results are discussed. Finally, the model is inverted for the entire ice sheet and the resulting estimates of geophysical surface properties are discussed.

\section{BACKGROUND}

This study employs data from the C-band European Remote-sensing Satellite (ERS) advanced microwave instrument (AMI) in wind scatterometer mode (hereafter referred to as simply ERS) which measures $\sigma^{\circ}$ at vertical polarization (Attema, 1991). ERS measurements span incidence angles from approximately $20^{\circ}$ to $60^{\circ}$. ERS has a fan-beam design with three fixed antennas, each measuring $\sigma^{\circ}$ at a different azimuth angle. Combining ascending and descending passes, ERS provides azimuth sampling at approximately six distinct azimuth angles at any given location. Azimuth sampling is fundamental to the primary purpose of ERS, which is to measure vector winds over the ocean. Wind retrieval is achieved by inverting an empirical model of the azimuth dependence of $\sigma^{\circ}$ as a function of wind speed and direction. We use the azimuth sampling of ERS to estimate parameters of a physical model of the azimuth dependence of $\sigma^{\circ}$ over Greenland. The data used in this study span a 30 day interval beginning Julian day (JD) 330 and ending JD 360 in 1996, which is during the winter when the backscatter of the Greenland surface is relatively constant.

Much of the analysis focuses on data from the Tunu- $\mathrm{N}$ $\left(78.0^{\circ} \mathrm{N}, 34.0^{\circ} \mathrm{W}\right)$ and NASA-U $\left(73.83^{\circ} \mathrm{N}, 49.5^{\circ} \mathrm{W}\right)$ sites (see Fig. 1). The raw data at each location comprise all ERS $\sigma^{\circ}$ measurements which lie within a $30 \mathrm{~km}$ radius of the location center. Figure 2 shows azimuth modulation observed in the raw data. The azimuth modulation at the Tunu- $\mathrm{N}$ site is over $3 \mathrm{~dB}$ peak-to-peak, and the modulation at the NASA-U site is about half this magnitude. 


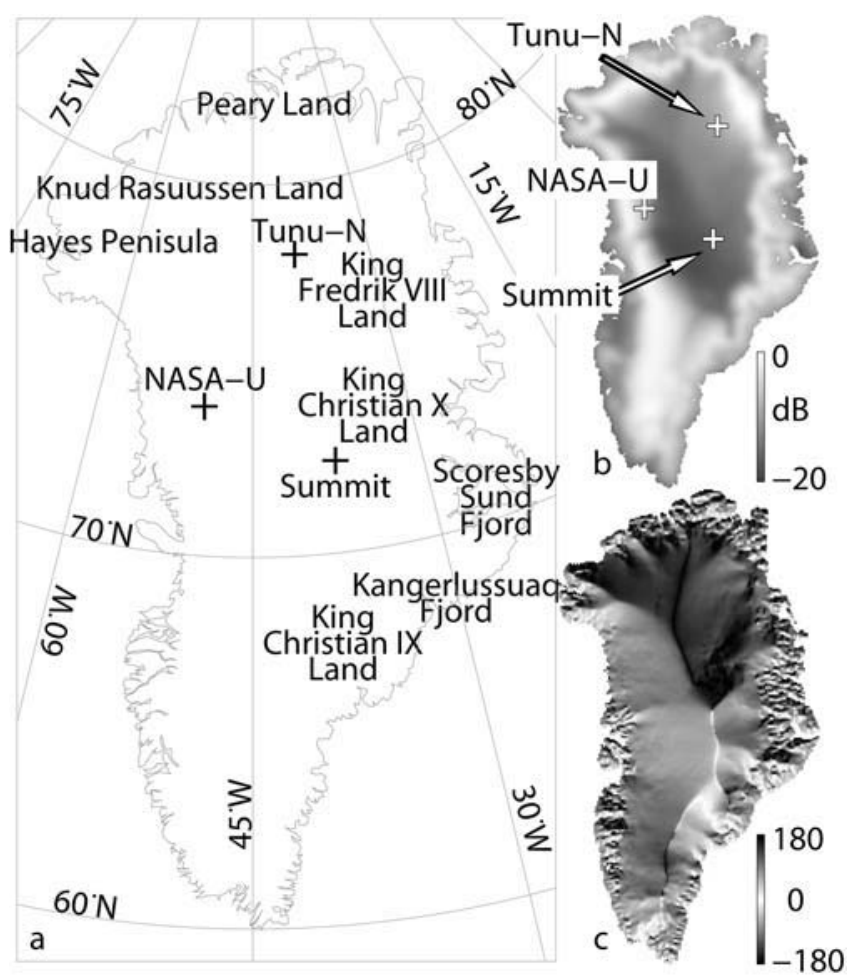

Fig. 1. (a) Map of Greenland. (b) ERS backscatter image showing $\sigma^{\circ}$ at $40^{\circ}$ incidence angle with the two study sites indicated. (c) Image of the direction of the gradient of the Greenland surface topography.

A review of the basic characteristics and geography of Greenland will aid this discussion. Greenland is almost completely covered by a thick layer of snow and ice. Near the summit, this layer is over $3 \mathrm{~km}$ deep (Fristrup, 1966). Because the ice is so deep, ground features are almost entirely masked out, leaving a surface with mountains present only on the periphery of the ice sheet (see Fig. 1c).

The Greenland ice sheet includes four diagenetic facies or zones (Benson, 1962; Fahnestock and others, 1993). The dry snow zone is the area where negligible melting occurs, and is located at the interior of the ice sheet. This region is characterized by low $\sigma^{\circ}$ values (Jezek and others, 1994; Long and Drinkwater, 1994). Because of small snow grains, the microwaves penetrate deep in the snow and are absorbed, producing little backscatter. Downslope from the dry snow zone lies the percolation zone which is characterized by high $\sigma^{\circ}$ values. The high backscatter is attributed to subsurface ice structures, termed ice pipes and ice lenses, which form when percolation channels in the wet snow freeze (Long and Drinkwater, 1994). Snow grains in the percolation facies firn are larger than snow grains in the dry snow zone due to growth associated with sublimation due to melt (Benson, 1962). The increased grain-size is another contributor to the increased backscatter found in this location (Long and Drinkwater, 1994). Further downslope is the wet or soaked zone where the previous years' accumulation becomes saturated with water during the summer melt. The backscatter in the soaked facies is relatively bright although it is of reduced intensity compared to the percolation zone. On the periphery of Greenland is the ablation zone where the annual snow accumulation completely melts each summer, leaving a surface of bare ice

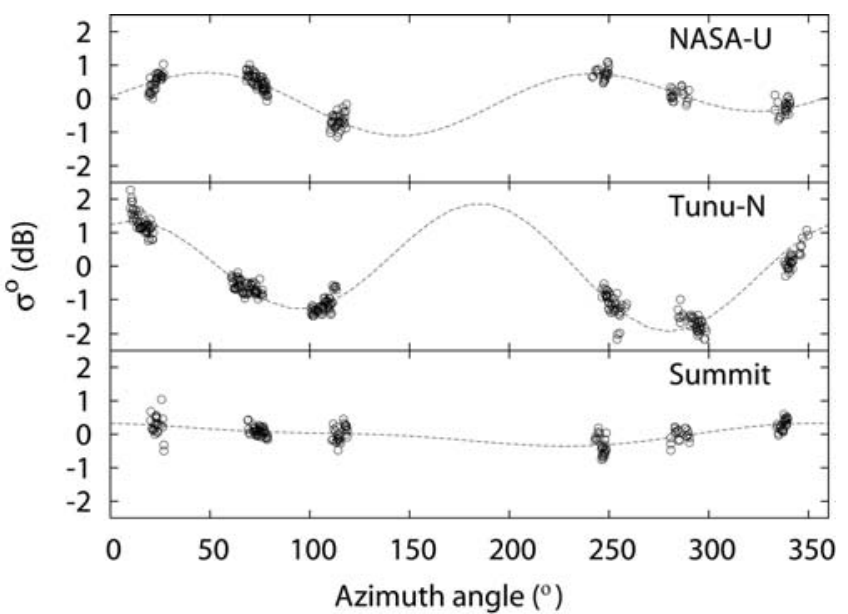

Fig. 2. Azimuth modulation observed in the ERS backscatter at three study locations (see Fig. 1). The circles represent the raw ERS measurements normalized to $40^{\circ}$ incidence angle and corrected for the spatial spread of the measurement centers using the empirical model in Ashcraft and Long (2005). The curves are second-order sinusoid fits to the data.

and rock. The backscatter in this region is of intermediate brightness and is dependent on season and location due to the non-uniformity of the surface.

Azimuth modulation of $\sigma^{\circ}$ has been observed previously over both Antarctica and Greenland. Over Antarctica, the orientation of the modulation is highly correlated with the wind direction (Ledroit and others, 1993; Young and others, 1996; Long and Drinkwater, 2000). Over Greenland, azimuth modulation of $\sigma^{\circ}$ was first observed by Swift and others (1985) at a location in the southern percolation zone. The modulation was observed in an aircraft banking maneuver during which the scatterometer remained focused on a specific location. The observed modulation is approximately $1 \mathrm{~dB}$ for $\sigma^{\circ}$ at Ku-band and is primarily first-order (a single cycle for $360^{\circ}$ ). Analysis by the authors indicates that the largest azimuth modulation at both C-band and Ku-band occurs in the dry snow of central and northern Greenland and is primarily second-order (Ashcraft and Long, 2005). The orientation of the azimuth modulation matches the general flow of the modeled wind fields of Bromwich and others (1996).

Over Antarctica, azimuth modulation of $\sigma^{\circ}$ is attributed to sastrugi (Ledroit and others, 1993; Rott and others, 1993; Rott and Rack, 1995; Young and others, 1996; Long and Drinkwater, 2000). Sastrugi are aligned such that the crest is parallel to the wind direction. They range in scale from a few to over a hundred meters (Bromwich and others, 1990). In this region, azimuth modulation is primarily second-order and the orientation is highly correlated with the prevalent wind direction (Long and Drinkwater, 2000).

Although azimuth modulation is attributed to sastrugi on the surface, there is a lack of physical models directly relating snow surface properties to azimuth modulation of $\sigma^{\circ}$. We propose a simple surface scattering model which relates azimuth modulation to physical surface properties. Our model assumes that sastrugi and other features of the same scale are the primary mechanism driving the modulation, which is consistent with second-order azimuth modulation such as that observed over much of the Greenland and Antarctica ice sheets. 


\section{SURFACE SCATTERING MODEL}

We propose a two-scale model to simulate the effects of sastrugi on $\sigma^{\circ}$ over Greenland. In our model, roughness on the order of a radar wavelength $(5.7 \mathrm{~cm})$ or smaller is termed 'small-scale' roughness. Roughness at scales from a few meters to a few kilometers is termed 'mesoscale' roughness. Features at this scale are larger than a wavelength and smaller than the dimensions of the measurement footprint. Features at scales larger than the size of the satellite footprint $(50 \mathrm{~km})$ are termed 'large-scale'. Roughness at scales in between these levels is assumed to be negligible. Our model surface is a composite of small-scale roughness and mesoscale roughness as illustrated in Figure 3 a.

While formulated as a surface scattering model, the scattering model represents an effective bulk equivalent to the actual multilayer surface including multilayer interaction and volume scattering, since the Greenland snowpack consists of multiple layers with each layer roughly equivalent to a year's accumulation (see Fig. 3b). Backscatter from internal layer boundaries may affect the model estimates of surface roughness. This effect is expected to be small for an individual layer because the dielectric difference between layers is small compared to the difference at the air/snow boundary. However, the combined contribution from multiple layers may be significant, especially for ERS which has a much larger penetration depth than Ku-band scatterometers. Though a simple physical model in the dry snow zone, we note that our model is not a complete physical description of the scattering processes in the percolation and ablation zones, where it should be considered an empirical model (cf. Rignot and others, 1993). Our model is primarily designed to help understand surface orientation.

Our model assumes the small-scale roughness is isotropic and is the primary factor in local incidence angle dependence of $\sigma^{\circ}$. The mesoscale roughness is assumed, in general, to be anisotropic, resulting in azimuth modulation of $\sigma^{\circ}$. To model the mesoscale roughness, the snow surface is viewed as a mesh of individual flat surfaces. The dimensions of each mesh element are assumed to be large compared with the incident wavelength. The satellite-observed large-scale backscatter $\left(\sigma_{\mathrm{ls}}^{\circ}\right)$ is the ensemble average of the small-scale backscatter $\left(\sigma_{\mathrm{ss}}^{\circ}\right)$ from the mesoscale mesh elements, i.e.

$$
\sigma_{\mathrm{ls}}^{\circ}(\theta, \phi)=\int_{0}^{\pi / 2} \sigma_{\mathrm{ss}}^{\circ}\left(\theta^{\prime}\right) P\left(\theta^{\prime} \mid \theta, \phi\right) \mathrm{d} \theta^{\prime},
$$

where $P\left(\theta^{\prime} \mid \theta, \phi\right)$ is the probability distribution of the local incidence angle. The local incidence angle distribution can be obtained from the surface slope distribution given the observation geometry. Because our model assumes that each mesh element is azimuthally isotropic, $\sigma_{\text {ss }}^{\circ}$ is only a function of the local incidence angle $\left(\theta^{\prime}\right)$.

To evaluate the contribution of the azimuth anisotropy in the mesoscale roughness, we compare our general model results with a form of the model constrained to be azimuthally isotropic in surface roughness. The general model for which the mesoscale surface roughness is anisotropic is termed the 'A-model'. The form of the model constrained to isotropic surface roughness is termed the 'I-model'. Additionally, we compare these two models with a form of the model where the mesoscale surface is constrained to be flat, which we term the 'F-model'. In the F-model $P\left(\theta^{\prime} \mid \theta, \phi\right)=\delta\left(\theta^{\prime}-\theta\right)$ and Equation (1) becomes $\sigma_{\mathrm{Is}}^{\circ}(\theta, \phi)=\sigma_{\mathrm{ss}}^{\circ}(\theta)$.
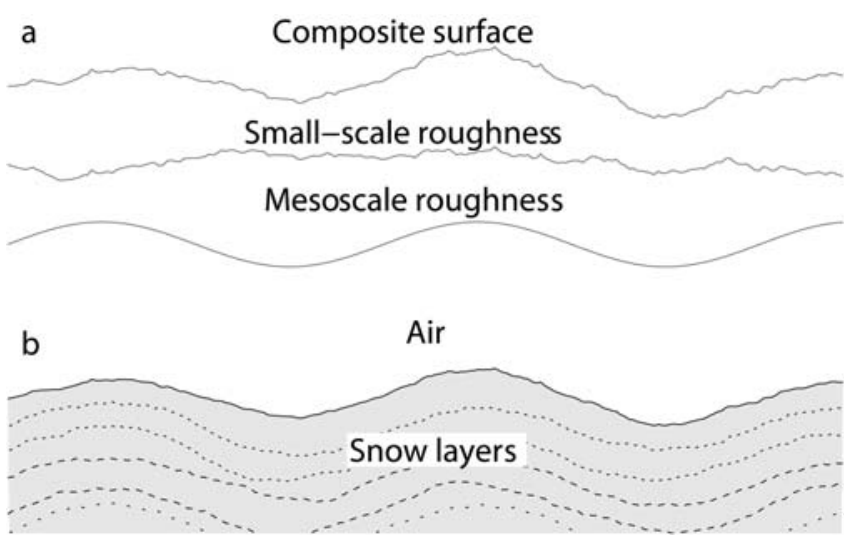

Fig. 3. (a) The snow surface is modeled as the composite of roughness at two scales: a mesoscale variation which is much larger than the electromagnetic wavelength, and a small-scale perturbation with variations on the order of a wavelength and smaller. (b) The actual surface includes multiple layers. The model represents the net response for both the surface and the interaction between multiple layers (see text).

\subsection{Mesoscale model}

The probability distribution of the local incidence angle, $P\left(\theta^{\prime} \mid \theta, \phi\right)$, is directly related to the mesoscale slope distribution $P(\vec{s})$, where $\vec{s}=\left[\begin{array}{ll}s_{X} & s_{y}\end{array}\right]^{\top}$ are the slopes in the $x$ and $y$ direction. Assuming that $\vec{s}$ is zero-mean and Gaussiandistributed,

$$
P(\vec{s})=\frac{1}{2 \pi|R|^{1 / 2}} \mathrm{e}^{-(1 / 2) \vec{s}^{\top} R^{-1} \vec{s}},
$$

where $R$ is the covariance matrix. The surface slope covariance, $R$, has a physical interpretation in terms of a root-mean-square (rms) slope as a function of azimuth. The singular value decomposition

$$
R=U \Sigma U^{\top}
$$

provides estimates of the minimum and maximum onedimensional rms slope and the associated azimuth angles. Let

$$
\Sigma=\left[\begin{array}{cc}
\xi_{1}^{2} & 0 \\
0 & \xi_{2}^{2}
\end{array}\right]
$$

and

$$
U=\left[\vec{u}_{1} \mid \vec{u}_{2}\right] .
$$

Then $\xi_{1}$ is the maximum one-dimensional rms surface slope, which is in the direction of $\vec{u}_{1}$, and $\xi_{2}$ is the minimum onedimensional rms surface slope, which is in the $\vec{u}_{2}$ direction. For the case of the I-model, $R$ is constrained to the form $R=\xi l$.

The simplified slope probability distribution in Equation (2) is related to the size and orientation of sastrugi over a snowfield. The distribution defines how the rms slope of a cross-section of the surface varies with direction. Over sastrugi, the rms slope is expected to be a maximum when the cross-section is orthogonal to the crests of the sastrugi, and minimum when the cross-section is parallel to the sastrugi crests. Because the crests of the sastrugi are aligned with the wind direction (Bromwich and others, 1990), $\vec{u}_{1}$ coincides with the cross-wind direction and $\vec{u}_{2}$ coincides with the up-/downwind direction. 


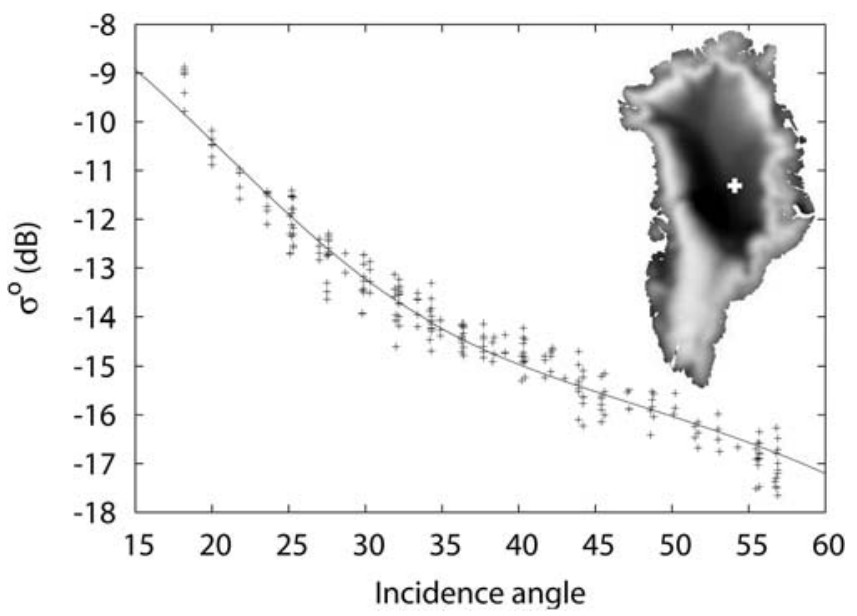

Fig. 4. A least-squares fit of the small-scale model to ERS data near the summit $\left(73.25^{\circ} \mathrm{N}, 37.28^{\circ} \mathrm{W}\right)$. At this location, the observed azimuth modulation in the ERS data is negligible.

\subsection{Small-scale model}

The other surface property required to evaluate Equation (1) is the small-scale dependence of $\sigma^{\circ}$ on incidence angle $\left(\sigma_{\mathrm{ss}}^{\circ}\left(\theta^{\prime}\right)\right)$. Backscatter over snow is a combination of both volume and surface scattering. Near nadir, surface scattering dominates, while at larger incidence angles volume scattering has a more significant contribution. The total backscatter at any incidence angle is the sum of these two components, i.e.

$$
\sigma_{\text {ss }}^{\circ}\left(\theta^{\prime}\right)=\sigma_{\text {surface }}^{\circ}\left(\theta^{\prime}\right)+\sigma_{\text {volume }}^{\circ}\left(\theta^{\prime}\right) .
$$

We use the small-perturbation method (SPM) to model surface scatter, which is appropriate for roughness scales of the order of the incident wavelength or smaller. SPM is also used by Ledroit and others (1993) in modeling backscatter over the Antarctic ice sheet. There are two key differences between our implementation of SPM and that of Ledroit and others. First, Ledroit and others assume an azimuthdependent form of SPM to account for azimuth modulation. However, SPM is only valid for roughness smaller than the incident wavelength $(5.7 \mathrm{~cm}$ for ERS). We assume the surface is isotropic at the SPM scale and that azimuth dependency is caused by surface tilt due to mesoscale features. This is consistent with the theory that sastrugi are the cause of azimuth modulation. Second, we assume a Gaussian surface correlation function as opposed to the exponential correlation function used by Ledroit and others. This is done because the Gaussian correlation function results in model estimates more consistent with the $\sigma^{\circ}$ measurements.

For SPM (Ulaby and others, 1986a)

$$
\sigma_{\text {surface }}^{\circ}\left(\theta^{\prime}\right)=8 k^{4} \sigma^{2} \cos ^{4} \theta^{\prime}\left|\alpha_{p p}\left(\theta^{\prime}\right)\right|^{2} W\left(2 k \sin \theta^{\prime}\right),
$$

where $k$ is the wavenumber in free space, $\sigma$ is the surface rms height and $W\left(2 k \sin \theta^{\prime}\right)$ is the power spectrum of the surface. The $\alpha_{p p}$ term is dependent upon polarization $(p)$. For the vertical-vertical ERS polarization (Ulaby and others, 1986a)

$$
\alpha_{\mathrm{vv}}\left(\theta^{\prime}\right)=\left(\varepsilon_{\mathrm{r}}-1\right) \frac{\sin ^{2} \theta^{\prime}-\varepsilon_{\mathrm{r}}\left(1+\sin ^{2} \theta^{\prime}\right)}{\left[\varepsilon_{\mathrm{r}} \cos \theta^{\prime}+\left(\varepsilon_{\mathrm{r}}-\sin ^{2} \theta^{\prime}\right)^{1 / 2}\right]^{2}},
$$

where $\varepsilon_{\mathrm{r}}$ is the relative dielectric constant of the surface.
Assuming a Gaussian correlation coefficient, the roughness spectrum is (Ulaby and others, 1986a)

$$
W\left(2 k \sin \theta^{\prime}\right)=\frac{1}{2} l^{2} \mathrm{e}^{-\left(k l \sin \theta^{\prime}\right)^{2}},
$$

where $I$ is the surface correlation length.

For the volume scattering model, we use (Swift, 1997)

$$
\sigma_{\text {volume }}^{o}\left(\theta^{\prime}\right)=T^{2}\left(\theta^{\prime}\right) \frac{n \sigma_{\mathrm{b}}}{2 \alpha} \cos \theta^{\prime},
$$

where $T\left(\theta^{\prime}\right)$ is the plane-wave power transmission coefficient, $n$ is the number density of scatters per unit volume, $\sigma_{\mathrm{b}}$ is the radar cross-section of a single scatter, and $\alpha$ is the bulk volume attenuation coefficient. Combining Equations (6), (7), (9) and (10) results in

$$
\begin{aligned}
\sigma_{\mathrm{ss}}^{\circ}\left(\theta^{\prime}\right)= & 4 k^{4} \sigma^{2} l^{2} \cos ^{4} \theta^{\prime}\left|\alpha_{p p}\left(\theta^{\prime}\right)\right|^{2} \mathrm{e}^{-\left(k / \sin \theta^{\prime}\right)^{2}} \\
& +T^{2}\left(\theta^{\prime}\right) \frac{n \sigma_{\mathrm{b}}}{2 \alpha} \cos \theta^{\prime} .
\end{aligned}
$$

The performance of Equation (11) in matching the ERS measurements is evaluated by fitting the model to the ERS data at a location where the surface is relatively flat and minimal azimuth modulation is observed. Figure 4 shows a least-squares fit of Equation (11) to ERS data from $73.25^{\circ} \mathrm{N}$, $37.28^{\circ} \mathrm{W}$, which is near the summit where the azimuth variation in $\sigma^{\circ}$ is negligible. The method used to obtain the least-squares error model estimates is described in section 3.3. Model roughness estimates for this location are $k l=3.22$ and $k \sigma=0.498$. The upper limit for the valid region of SPM is $k \sigma<0.3$ (Ulaby and others, 1986a). Because the estimate obtained for $k \sigma$ is somewhat larger than this limit, care must be used when interpreting these parameters in terms of actual surface roughness values. However, as observed in Figure 4, the model provides a good fit to the data and thus is useful as an empirical model describing the local incidence angle dependence of $\sigma^{\circ}$. Also, as discussed at the beginning of section 3, Equation (11) is a bulk equivalent model representing the snowpack, which consists of many layers, as one single layer. Therefore, the surface roughness parameters represent an equivalent single layer roughness estimate for a multilayered surface. The small-slope approximation (SSA) is a possible alternative to SPM in future studies, as SSA is valid for larger roughness scales (Broschat and Thorsos, 1997). However, SSA is not used in this study because it adds significant complexity to the model and the model inversion process. The quality of the fit and the simplicity provided by the SPM model is deemed appropriate for this initial study.

\subsection{Model inversion}

Estimation of surface parameters from $\sigma^{\circ}$ measurements requires inversion of the surface model. The model inversion is obtained by minimizing the rms error

$$
\chi=\left[\left(\vec{\sigma}^{\circ}-\vec{\sigma}_{\mathrm{ls}}^{\circ}\right)^{\top}\left(\vec{\sigma}^{\circ}-\vec{\sigma}_{\mathrm{ls}}^{\circ}\right)\right]^{1 / 2},
$$

where $\vec{\sigma}^{\circ}$ is a vector of the measurement values and $\vec{\sigma}_{\mathrm{ls}}^{\circ}$ is a vector of the model estimates. The minimization of Equation (12) with respect to the model parameters is a secondorder non-linear regression problem. We solve it using the iterative Levenberg-Marquardt technique (Marquardt, 1963). On the base level, the small-scale model parameters which minimize Equation (12) are estimated for given $P\left(\theta^{\prime} \mid \theta, \phi\right)$ which is obtained from a specified mesoscale 
slope covariance, $R$. This process is iterated for different $R$ values until the minimum of Equation (12) is obtained.

The distribution $P\left(\theta^{\prime} \mid \theta, \phi\right)$ is different for each measurement based on the measurement geometry. For each measurement, the calculation of $P\left(\theta^{\prime} \mid \theta, \phi\right)$ from the surface slope covariance, $R$, is performed numerically. This is done by discretizing the range of surface slopes along with the associated probability for each slope combination. Each slope combination $\vec{S}$ is represented by a unit vector $\hat{\mathbf{z}}^{\prime}$ orthogonal to the local surface. The corresponding local incidence angle is $\theta^{\prime}=\cos ^{-1}\left(\hat{\mathbf{z}}^{\prime} \cdot \hat{\mathbf{r}}\right)$, where $\hat{\mathbf{r}}$ is a unit vector in the $\theta, \phi$ direction. Each surface slope combination is binned according to the local incidence angle, $\theta^{\prime}$, to obtain a discrete estimate of the local incidence angle distribution $P\left(\theta^{\prime} \mid \theta, \phi\right)$.

Given $P\left(\theta^{\prime} \mid \theta, \phi\right)$, the small-scale surface parameters which minimize Equation (12) are obtained. To solve for the small-scale model parameters, Equation (11) is rewritten as

$$
\sigma_{\mathrm{sS}}^{\circ}\left(\theta^{\prime}\right)=P \cos ^{4} \theta^{\prime}\left|\alpha_{p p}\left(\theta^{\prime}\right)\right|^{2} \mathrm{e}^{-Q \sin ^{2} \theta^{\prime}}+T^{2}\left(\theta^{\prime}\right) V \cos \left(\theta^{\prime}\right),
$$

where the parameters $P=4 k^{4} \sigma^{2} l^{2}, Q=k^{2} l^{2}$ and $V=$ $n \sigma_{\mathrm{b}} / 2 \alpha$, are used as the unknown parameters. To obtain $\alpha_{p p}$ and $T\left(\theta^{\prime}\right)$, we set $\varepsilon_{\mathrm{r}}=1.7$, corresponding to a snow density of $0.38 \mathrm{~g} \mathrm{~cm}^{-3}$ (Ulaby and others, 1986b, p. 2061) which is typical of the densities observed by Benson (1962, p. 66) in the upper percolation facies.

\subsection{Results}

The fit of the surface scattering model to the ERS $\sigma^{\circ}$ measurements is analyzed using two study areas. As mentioned in section 2, the study areas are centered at the Tunu- $\mathrm{N}$ and NASA- $U$ sites to enable comparison between Greenland Climate Network automatic weather station (AWS) in situ wind measurements and model-produced preferential directions in the surface roughness. Although the measurement centers of the aquired data are within $30 \mathrm{~km}$ of each of the study locations, the distance and the direction of the measurement from the study point can significantly affect the magnitude of the backscatter. This effect is minimized prior to model inversion by normalizing the data to match the study point using a method based on the spatial gradient of the backscatter at each location as discussed in Ashcraft and Long (2005).

Estimates obtained through inverting the A-model provide a good fit to the data. Plots comparing A-model estimates to measurements are shown in Figure 5. The model estimates show peak-to-peak azimuth modulation of over $3 \mathrm{~dB}$ at Tunu-N and up to $2 \mathrm{~dB}$ at NASA-U. At Tunu-N, the variability of the data around the model estimates (which includes variations due to incidence angle) is generally smaller than $1 \mathrm{~dB}$. The data are centered at the model estimate, indicating a good fit. At the NASA-U location, the spread in the data at each azimuth is larger, in many cases over $2 \mathrm{~dB}$, and not as consistently centered around the estimate. This apparent discrepancy is attributed in part to the incidence angle dependence of $\sigma^{\circ}$, which causes up to $1.5 \mathrm{~dB}$ of variation in the $5^{\circ}$ incidence angle range for each sub-plot (see Fig. 5d).

Further plots for evaluating the fit of the model estimates to the data are provided in Figure $5 \mathrm{c}$ and $\mathrm{d}$. These show the incidence angle dependence of the model at two azimuth angles along with $\sigma^{\circ}$ measurements with azimuth angles
Table 1. Maximum $\left(\xi_{1}\right)$ and minimum $\left(\xi_{2}\right)$ one-dimensional rms mesoscale surface slopes $\left(\mathrm{m} \mathrm{m}^{-1}\right)$ and corresponding azimuth angles (degrees relative to north) obtained from the A-model surface slope distribution estimates at the two study locations. Because the model makes no distinction between up- and downwind, there is a $180^{\circ}$ ambiguity in the direction of $\vec{u}_{2}$. The displayed value is the ambiguity closest to the measured wind-flow direction. Also included is an estimate of the mesoscale rms surface slope for the I-model

\begin{tabular}{lccccc}
\hline \multicolumn{9}{c}{ A-model } & & \multirow{2}{*}{ I-model } \\
& $\xi_{1}$ & $\xi_{2}$ & $\angle \vec{u}_{1}$ & $\angle \vec{u}_{2}$ & $\xi$ \\
\hline Tunu-N & 0.29 & 0.12 & $193^{\circ}$ & $103^{\circ}$ & 0.055 \\
NASA-U & 0.21 & 0.11 & $46^{\circ}$ & $316^{\circ}$ & 0.050 \\
\hline
\end{tabular}

within $\pm 10^{\circ}$. Here the incidence angle dependence of $\sigma^{\circ}$ is distinctly different for the displayed azimuth ranges. At Tunu- $\mathrm{N}$, the $\sigma^{\circ}$ measurements around $\phi=15^{\circ}$ are consistently about $3 \mathrm{~dB}$ larger than the case where $\phi=290^{\circ}$. This behavior is matched by the model output. At NASA-U, the model estimates a $2 \mathrm{~dB}$ bias in the mean backscatter due to azimuth angle, with the larger intensities at $\phi=245^{\circ}$ and the smaller intensities at $\phi=115^{\circ}$. The model-estimated bias is slightly larger than the spread observed in the measurements.

The performance of the A-model is evaluated by comparing the rms of the residuals (Equation (12)) with that of the I-model and the F-model. The A-model performs similarly at both study locations, with rms residual errors of around $0.4 \mathrm{~dB}$. The rms errors are significantly larger for the other two models: $1.05 \mathrm{~dB}$ at Tunu- $\mathrm{N}$ and $0.65 \mathrm{~dB}$ at NASA$U$. We note that ERS measurement accuracy is approximately $0.2 \mathrm{~dB}$ (Attema and Lecomte, 1998).

The A-model enables estimation of the directional dependence of the mesoscale surface slopes. The correlation matrix of the mesoscale surface slope distribution indicates how the one-dimensional rms surface slope varies with azimuth angle as discussed in section 3.1. Recall that $\xi_{1}$ and $\xi_{2}$ are estimates of the maximum and minimum onedimensional rms surface slope, and the corresponding azimuth angles are given by the orientations of $\vec{u}_{1}$ and $\vec{u}_{2}$. Estimates of these values for the two study locations are listed in Table 1. The rms surface slope in the dominant roughness direction $\left(\xi_{1}\right)$ is largest at Tunu-N, indicating steeper mesoscale surface slopes at this location. This is attributed to the difference in accumulation rates rather than a difference in wind speed, since the speeds shown in Figure 5 exhibit no significant difference in magnitude between the two locations. At Tunu- $\mathrm{N}$ the accumulation rate is less than one-half the accumulation rate at the NASA-U site (Ohmura and Reeh, 1991; Bales and others, 2001). The accumulation rate contributes to the differences in the thickness of the annual layers, the snow density and other properties. Unfortunately, in situ data are not available to validate the observed rms surface slopes.

The plots at the bottom of Figure 5 show the distribution of wind speed and direction during 1996 at the two locations. The model estimates show that the minimum of the azimuth modulation is in the $\vec{u}_{2}$ direction. As discussed in section 3.1, this corresponds with the up-/downwind direction. This relationship between the dominant wind 

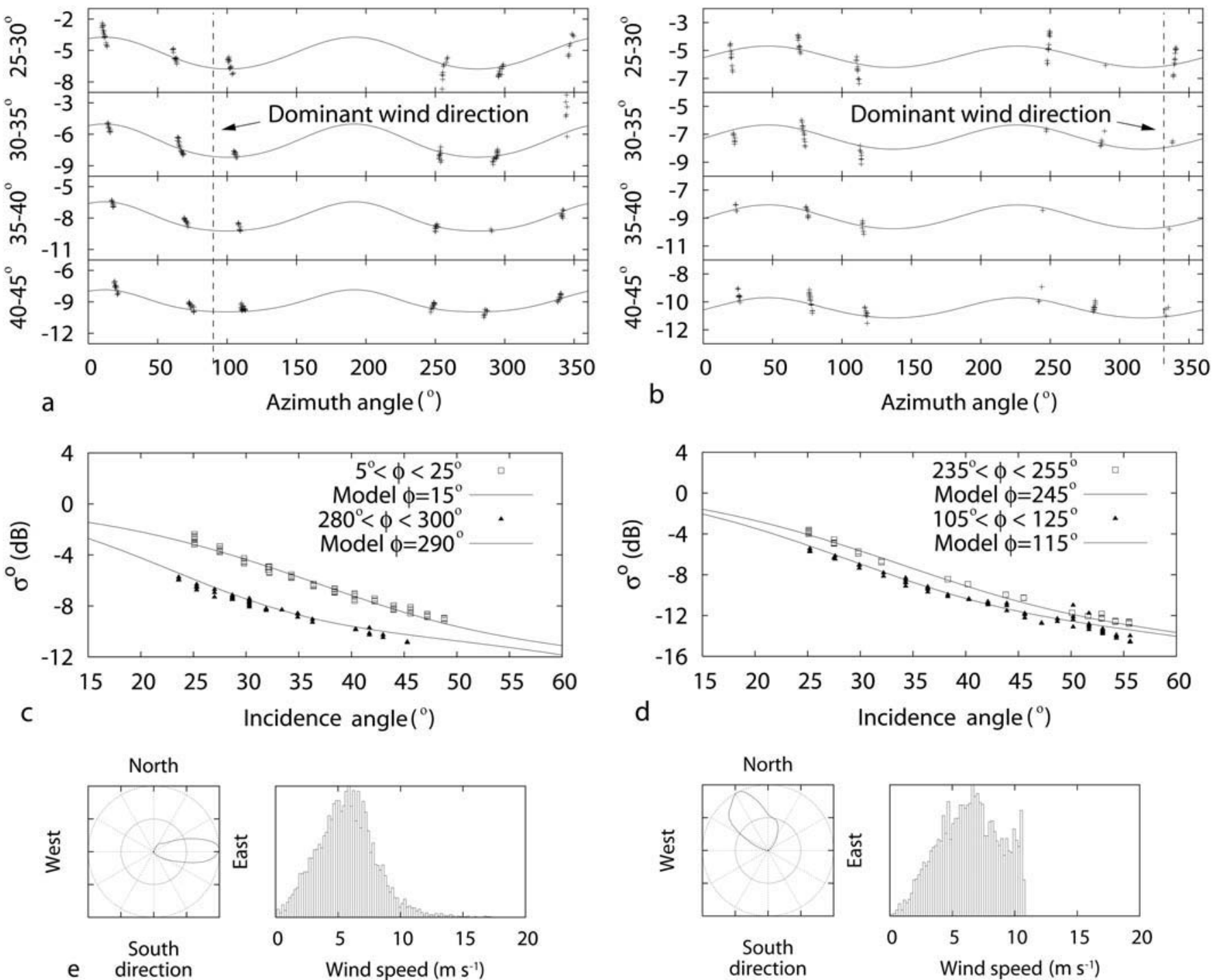

Fig. 5. Illustration of the A-model fit to ERS measurements at the Tunu-N $\left(78.0^{\circ} \mathrm{N}, 34.0^{\circ} \mathrm{W}\right)(\mathrm{a}, \mathrm{c}, \mathrm{e})$ and $\mathrm{NASA}-\mathrm{U}\left(73.83^{\circ} \mathrm{N}, 49.5^{\circ} \mathrm{W}\right)(\mathrm{b}, \mathrm{d}, \mathrm{f})$ sites (see Fig. 1). (a, b) The plots are divided into four incidence angle bins with the range of each bin indicated on the vertical axis. The raw ERS measurements from each bin are indicated by ' + ' symbols. Much of the vertical spread observed in the raw data at each azimuth angle is due to the variation in the measurement incidence angles which is not compensated for here. The curves represent the model fit to the raw data where $\theta$ is set to the center of the respective incidence angle range and $\phi$ varies along the horizontal axis. (c, d) The plots show the incidence angle dependence of the raw data and the surface model fit. For each plot, raw ERS data from two ranges of $\phi$ are shown with the range indicated in the key. The curve shows the A-model where $\phi$ is set to the center of each range indicated in the key and $\theta$ varies along the horizontal axis. (e, f) The plots show the wind statistics at each location during 1996 obtained from the Greenland Climate Network (Steffen and others, 1996). The left plot is a circular histogram of the wind source direction while the right plot is a histogram of the wind speed.

direction and the minimum of the azimuth modulation is consistent with the results of Long and Drinkwater (2000) over Antarctica. At both locations the orientation of $\vec{u}_{2}$ is within $15^{\circ}$ of the dominant wind direction shown in Figure 5 .

The small-scale parameter estimates are listed for each of the three models in Table 2. Because the estimates of $k \sigma$ are outside the valid range for SPM, as discussed in section 3.2 the parameters should not be interpreted as the actual surface roughness. However, the model estimates are still valuable for gaining insights into the scattering at these locations. Inspection of the two terms in Equation (11) reveals important differences in the contribution of each small-scale parameter to the overall backscatter.

The combination of surface and volume scattering dictates the backscatter model dependence on incidence angle. In general, surface scattering generates a steeper incidence angle dependence at large incidence angles than does volume scattering. The large estimates of $k \sigma$ cause the surface scattering term to contribute more at large incidence angles, resulting in a steeper incidence angle dependence than is provided by the volume scattering term only. Thus, to adequately model the surface in terms of snow properties, either a more complex volume scattering term having a steeper roll-off with incidence angle or a surface scattering term valid for larger estimates of the backscatter at high incidence angles is required. An alternative approach for future studies is a multilayer model combining the surface and volume scattering from the individual layers. However, such a model will increase the number of unknown parameters, decreasing the probability for well-conditioned model inversion. 


\subsection{Ice-sheet-wide surface parameter estimation}

\subsubsection{Mesoscale parameters}

We have inverted the three variations of the surface scattering model to obtain parameter estimates across the ice sheet. Estimates of the mesoscale slope distribution parameters are shown in Figure 6. Recall that $\xi_{1}$ and $\xi_{2}$ are the square roots of the singular values of the correlation matrix, $R$, from the A-model and provide estimates of the maximum and minimum one-dimensional rms surface slopes. Perhaps the most compelling indicator of the validity of the A-model and the assumption that wind-formed sastrugi are the dominant mechanism driving azimuth modulation over Greenland is found in the orientation of $\vec{u}_{2}$. As discussed previously, over a field of sastrugi $\vec{u}_{2}$ is in the up-/downwind direction. Figure 6c shows streamlines of $\left\langle\vec{u}_{2}\right.$ (where indicates the vector) imposed over an image of $\xi_{1}$. The streamlines are very similar to the katabatic surface wind fields modeled by Bromwich and others (1996) over the Greenland ice sheet. One of the most identifiable features in both the $\left\langle\vec{u}_{2}\right.$ streamlines and the Bromwich wind field is the wind divergence region running from the summit northwest to Hayes Peninsula (see Fig. 1). Over the southern portion of the ice sheet, the dominant wind direction also matches $\angle \vec{u}_{2}$. The lack of swath patterns in this and the other parameter images is a strong indicator that physical properties of the surface are being observed, and that the variations in the model parameter estimates are not due to instrument effects.

Features of the wind pattern are evident throughout the dry snow zone in the $\xi_{1}$ image. Locations with divergent wind fields give small estimates of the sastrugi slopes, which are consistent with the low wind speeds and correspondingly small sastrugi found there. This is most apparent where the wind field diverges northwest of the summit. It is also observed on the east of Greenland between the Scoresby Sund and Kangerlussuaq Fjords (see Fig. 1a) where Bromwich and others (1996) estimate corresponding low wind speeds. Along the ridge separating these two fjords, the mean wind field diverges. Thus low winds are expected on the ridge and high winds on the slopes leading down to the fjords. This is observed in the $\xi_{1}$ image as a dark region (the ridge) between two brighter regions. Another pronounced feature in the $\xi_{1}$ image is an area of low estimates reaching almost directly north of the summit to the edge of the ice sheet between King Fredrik VIII Land and King Christian X Land. The location of these low estimates corresponds to the windward side of a ridge running northwest from the summit observed in Figure 1b. For katabatic winds, such as those modeled by Bromwich and others (1996), the wind flow is driven by gravity and local topography. Katabatic cooling increases the air density so

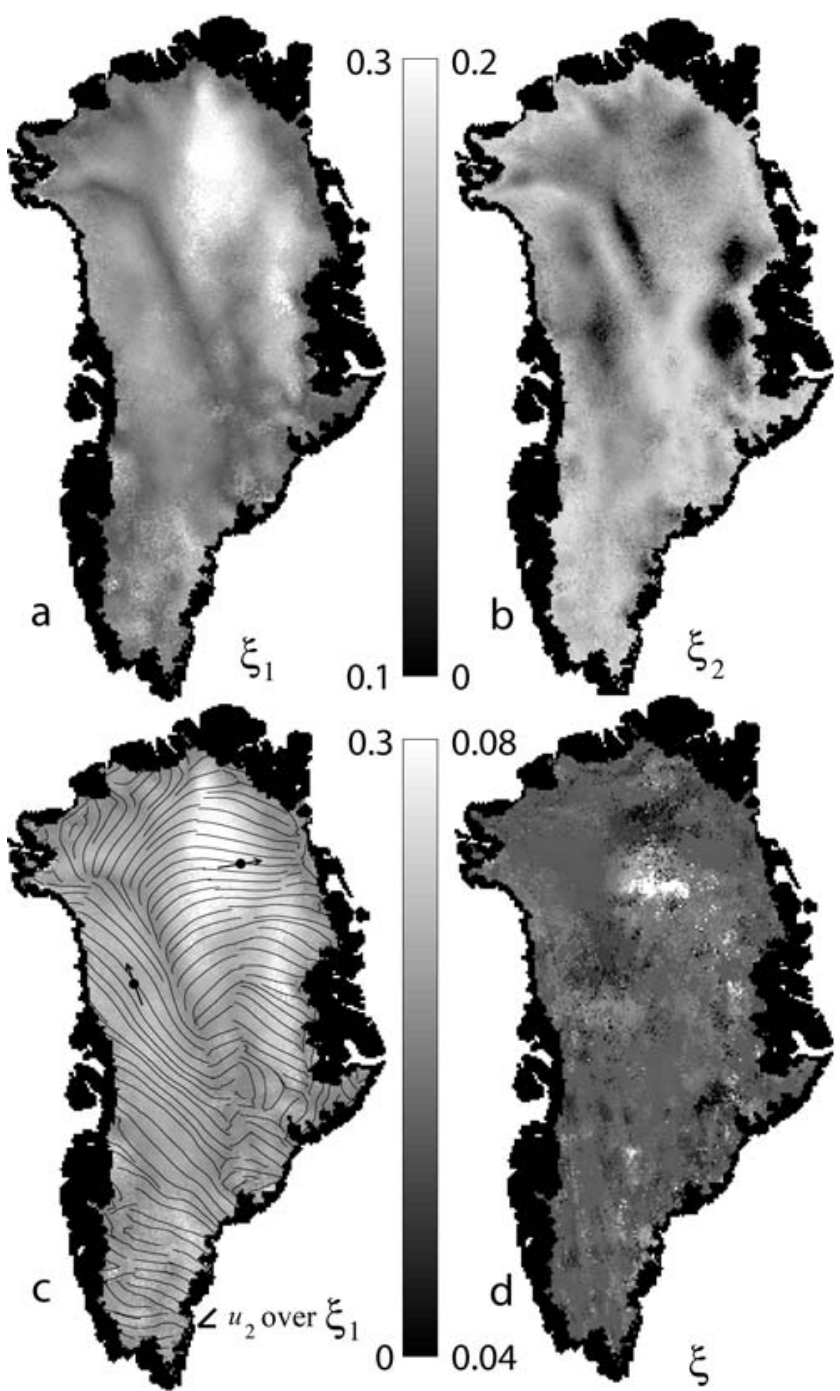

Fig. 6. Images showing estimates of the mesoscale slope distribution correlation matrix singular values. $(\mathrm{a}, \mathrm{b})$ Maximum $\left(\xi_{1}\right)$ and minimum $\left(\xi_{2}\right)$ one-dimensional rms surface slopes estimated using the A-model. (c) Streamlines of the A-model estimate of the wind flow $\left(\vec{u}_{2}\right.$ direction) imposed over a $\xi_{1}$ image. Direction is not indicated due to the $180^{\circ}$ ambiguity in the model. Arrows indicate AWS-measured average wind-flow direction during 1996. (d) Rms surface slope obtained using the I-model.

that it sinks downslope. In the region north of the summit with low rms surface slope estimates, the wind and the topographical slope are nearly orthogonal. Because gravity is not accelerating the wind in the dominant flow direction, lower wind speeds are expected. This is consistent with the low $\xi_{1}$ estimates. One possible contributor to the differences

Table 2. Small-scale parameters for the three surface scattering models at the two study locations where $V=n \sigma_{\mathrm{b}} / 2 \alpha$. Each parameter is discussed in detail in section 3.2

\begin{tabular}{|c|c|c|c|c|c|c|c|c|c|}
\hline & \multirow{3}{*}{$k \sigma$} & \multicolumn{2}{|c|}{ A-model } & \multicolumn{3}{|c|}{ I-model } & \multicolumn{3}{|c|}{ F-model } \\
\hline & & $k l$ & V & $k \sigma$ & $k l$ & $V$ & $k \sigma$ & $k l$ & $V$ \\
\hline & & & $\mathrm{dB}$ & & & $\mathrm{dB}$ & & & $\mathrm{dB}$ \\
\hline Tunu-N & 1.24 & 3.62 & -8.8 & 1.17 & 2.87 & -8.7 & 1.18 & 2.82 & -8.7 \\
\hline NASA-U & 1.35 & 3.15 & -11.2 & 1.39 & 2.76 & -11.2 & 1.40 & 2.72 & -11.2 \\
\hline
\end{tabular}




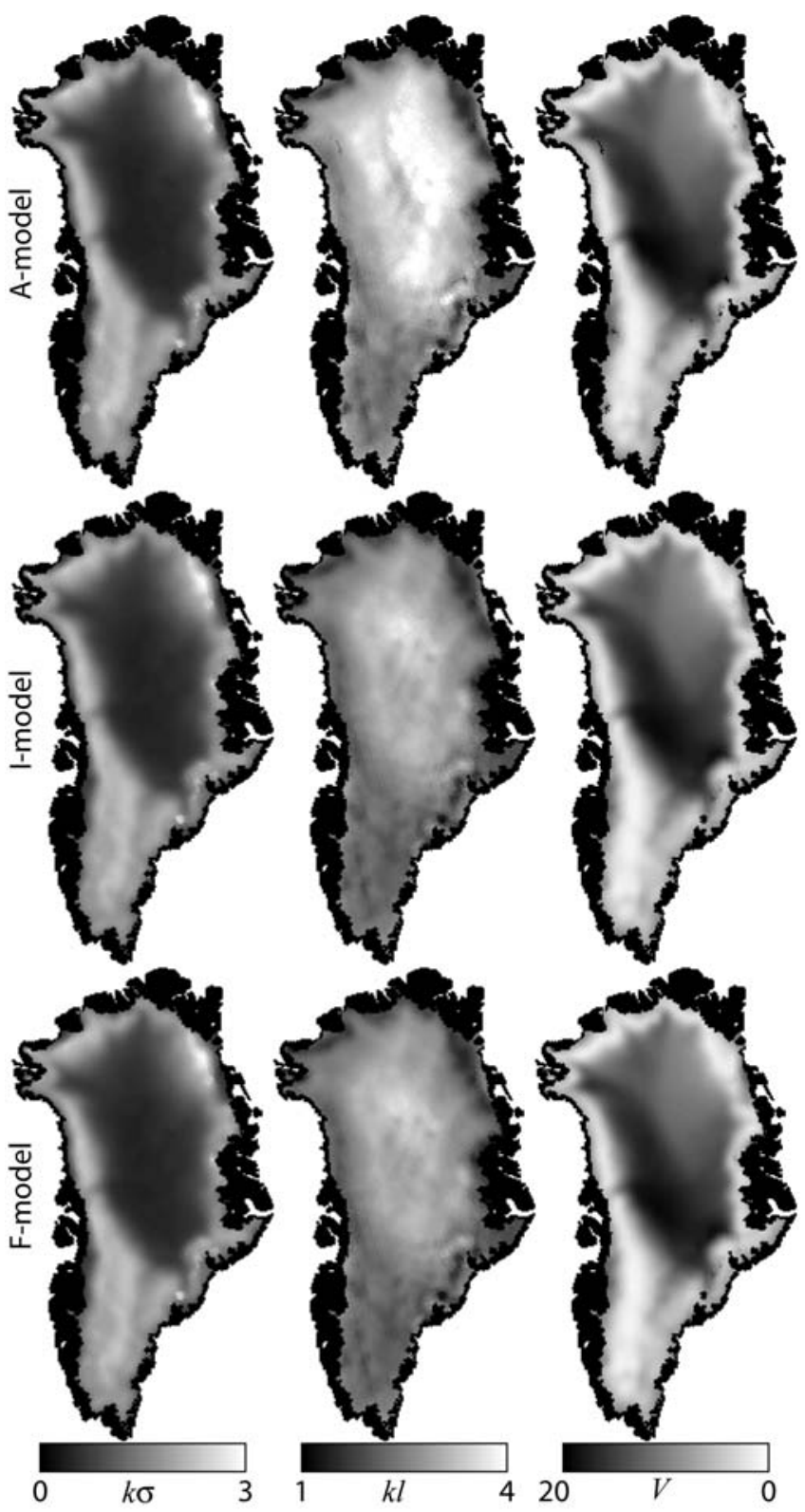

Fig. 7. Images of the estimated small-scale surface model parameters across the ice sheet estimated for the three models from ERS $\sigma^{\circ}$ measurements.

in the $\xi_{1}$ estimates in the east and west dry snow zones is the difference in the accumulation rates. The northeast dry snow zone has about half the accumulation rate of the west dry snow zone (Ohmura and Reeh, 1991; Bales and others, 2001).

The low estimates of $\xi_{1}$ in the percolation zone are attributed to changes in the snowpack associated with melt. Recall that the percolation zone is the region where melt occurs, but the snowpack does not become saturated with liquid water. Melt significantly changes the backscatter properties of the surface such that after refreeze the backscatter is dominated by scattering from subsurface ice structures (Jezek and others, 1994) altering or masking the azimuth modulation signature. During the winter, scattering from the dry snow surface layer that has accumulated since the last melt is mitigated by its transparency and the strong backscatter from the subsurface percolation facies.

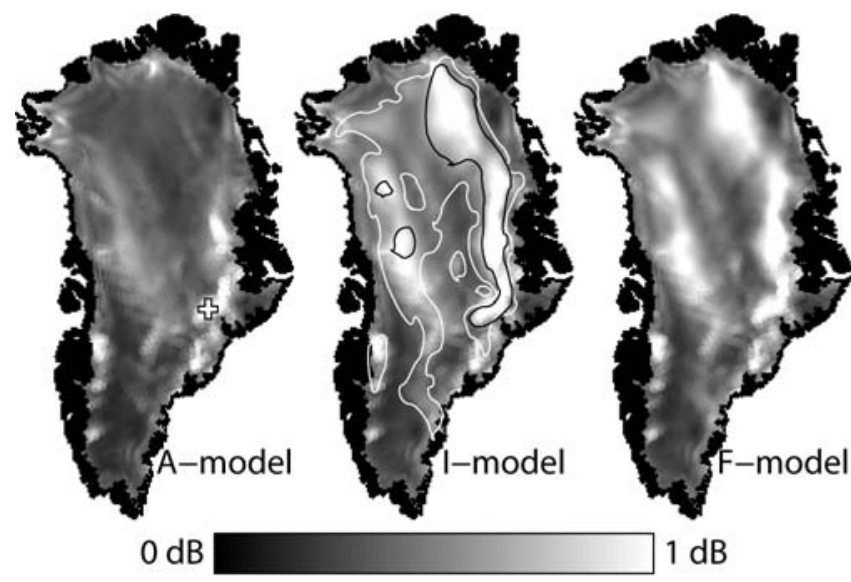

Fig. 8. Images of the rms error of the different models across the ice sheet. The A-model image includes a ' + ' mark indicating the location $\left(69.5^{\circ} \mathrm{N}, 34.3^{\circ} \mathrm{W}\right)$ which is used for further analysis of the rms error. The I-model image has a white outline around the regions where the observed azimuth modulation is larger than $1 \mathrm{~dB}$ peakto-peak and a black outline around the regions where the observed modulation is larger than $2 \mathrm{~dB}$.

Wind-field related features observed in the $\xi_{1}$ image are also apparent in the $\xi_{2}$ image. The estimates of $\xi_{2}$ at the divergent wind region between the summit and Hayes Peninsula are high while just outside the divergent region the estimates drop significantly. Low estimates are also found in other areas, mostly in the lower portions of the dry snow zone. These are areas where significant acceleration and narrow cross-wind variance is expected in the wind field as it nears the edge of the ice sheet where the surface slope is increasing. The ice-sheet slope is particularly large in King Christian X Land where the lowest estimates of $\xi_{2}$ are found.

\subsubsection{Small-scale parameters}

The images in Figure 7 show the model parameters for the local incidence angle dependence. The small-scale parameters are estimated using a least-squares approximation for the three models. The $k \sigma$ and $V$ terms primarily affect the magnitude of the $\sigma^{\circ}$ estimates, whereas the $k l$ term is a major contributor to the incidence angle dependence of the $\sigma^{\circ}$ estimates. The $k \sigma$ estimates are nearly identical for all three models, as are the $V$ estimates. The $k \sigma$ and $V$ images also exhibit very similar features. Three regions are observed in each image: a dark region at the interior surrounded by a bright region and then a reduced intensity region on the periphery. These regions roughly correspond to the dry snow, percolation and wet snow facies. In general, the magnitudes of $k \sigma$ and $V$ are correlated with the magnitude of the backscatter observed from the different facies. One feature observed only in the $V$ image is the division of the dry snow zone into the two distinct regions first noted by Long and Drinkwater (1994).

Correlation length $(k l)$ estimates are lowest in the percolation zone and highest in the dry snow zone. The I-model and F-model result in approximately the same $\mathrm{kl}$ estimates. The A-model is similar to the other two models in the percolation zone but produces significantly higher estimates of $k l$ in the dry snow zone which has the largest azimuth modulation. The higher estimates of $k l$ observed in this region indicate an increased dependence of $\sigma^{\circ}$ on incidence angle. 


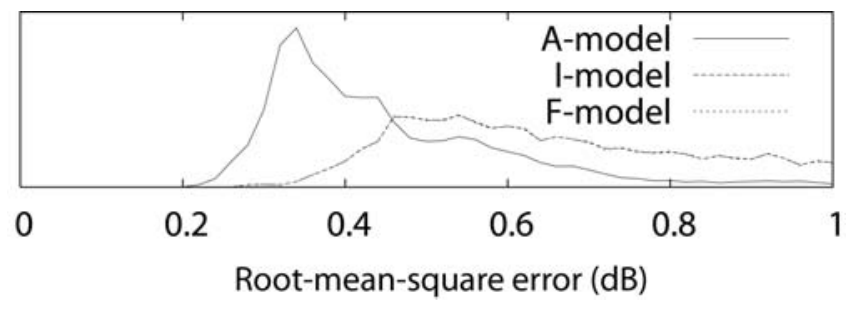

Fig. 9. Normalized histogram of the rms error for the different models in regions where the observed azimuth modulation is larger than $1 \mathrm{~dB}$ peak-to-peak. The I-model and F-model overlap, making them almost indistinguishable.

\subsubsection{Error analysis}

Figure 8 shows the rms error across the ice sheet for the three models, as defined by Equation (12). The A-model error is significantly smaller than that of the other two models throughout most of the dry snow zone, particularly in the northeast. The distribution of the rms error for each model across the ice sheet is shown in Figure 9. The A-model mean rms error is 0.46 compared to $0.56 \mathrm{~dB}$ for the $\mathrm{I}$ - and F-models. Over a significant portion of the ice sheet the A-model rms error approaches the ERS accuracy of $0.2 \mathrm{~dB}$ (Attema and Lecomte, 1998). When restricted to areas with observed azimuth modulation of over $1 \mathrm{~dB}$ peak-to-peak (see Fig. 8) the mean rms error of the A-model remains at $0.46 \mathrm{~dB}$ while the mean rms error of the other models increases to $0.70 \mathrm{~dB}$. When further restricted to areas with azimuth modulation of over $2 \mathrm{~dB}$ the mean rms error of the A-model increases slightly to $0.52 \mathrm{~dB}$ while the mean rms error of the other models jumps to $0.93 \mathrm{~dB}$.

Areas with above-average rms errors are observed between King Christian IX Land and King Christian X Land along the east edge of the dry snow zone. A close inspection of the $\sigma^{\circ}$ measurements in these large-error regions reveals anomalies in the data at varying azimuth and incidence angles. Figure 10 shows data from $69.5^{\circ} \mathrm{N}, 34.3^{\circ} \mathrm{W}$ (see Fig. 8), a location within this high-error region. From Figure 10a we observe that the largest errors occur around $\phi=75^{\circ}$. In Figure $10 \mathrm{~b}$ it appears that the incidence angle dependence around $\phi=75^{\circ}$ is reversed from the common incidence angle dependence observed over Greenland. At this location and azimuth angle, $\sigma^{\circ}$ increases with incidence angle as opposed to the decrease of $\sigma^{\circ}$ with incidence angle commonly observed over Greenland. Similar anomalies in the ERS measurements are observed throughout the regions with high rms errors. Between King Christian IX Land and King Christian $X$ Land the anomalies are generally restricted to the range $-60^{\circ}<\phi<100^{\circ}$, though the anomalies are not always a reversal in the incidence angle dependence as observed in Figure 10. In general, the anomalies occur as abnormally high or low $\sigma^{\circ}$ measurements within a small azimuth and incidence angle range. In order to understand the phenomena driving these anomalies, a more complete azimuth and incidence angle sampling of $\sigma^{\circ}$, combined with in situ data, is needed. It does not appear to be an instrument effect, as the specific azimuth and incidence angles where such anomalies are observed varies with location.

\section{SUMMARY}

Significant azimuth modulation occurs in the ERS $\sigma^{\circ}$ measurements of Greenland. This modulation is beneficial
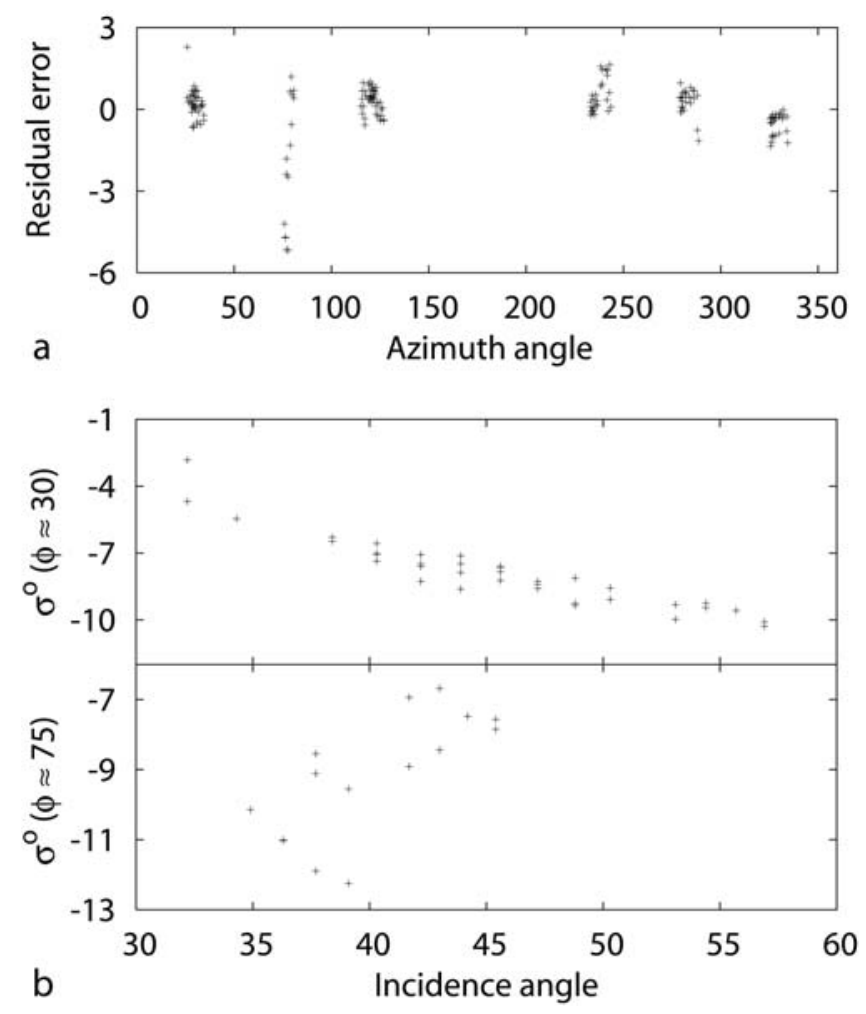

Fig. 10. ERS data and A-model estimate errors at $69.5^{\circ} \mathrm{N}, 34.3^{\circ} \mathrm{W}$ (see Fig. 8). (a) A-model estimation error vs azimuth angle. At azimuth angles near $75^{\circ}$ there is a high concentration of aboveaverage estimation errors. (b) Incidence angle dependence of raw $\sigma^{\circ}$ measurements at two azimuth angles. At $\approx 30^{\circ}$ (top) the measurements exhibit the expected fall-off with incidence angle. However, at $\approx 75^{\circ}$ the measurements exhibit a counter-intuitive increase with incidence angle.

for further understanding the properties of the ice sheet, with particular application to wind flow. A simple two-scale model can be used to model the combined incidence and azimuth angle dependence of the data. This model assumes that the primary mechanism driving the azimuth modulation is anisotropy in the mesoscale surface roughness. The smallscale incidence angle dependence is modeled using a combination of surface and volume scattering. Because the resulting surface roughness estimates are slightly outside the accepted range for the surface scattering model, the smallscale model must be viewed as an empirical model rather than a physical model. Future studies may implement the model using SSA which has a larger region of validity than SPM. However, SSA adds significantly to the complexity of the model.

The model is aimed at detecting surface orientations in the dry snow zone but can be applied elsewhere under appropriate conditions. Model estimates are consistent with the idea of wind-formed sastrugi playing a dominant role in the azimuth modulation. Estimates of the direction with the minimum one-dimensional rms slope are highly correlated with katabatic wind flow patterns and AWS measurements. Many features related to the wind flow are observed in the model estimates of the surface slope characteristics. The largest mesoscale slope estimates occur in the lower portions of the dry snow zone. Even in the percolation zone where azimuth modulation is small and scattering from subsurface ice structures is significant, model estimates of 
the surface roughness directionality are strongly correlated with katabatic wind patterns.

The A-model, which assumes anisotropic mesoscale surface roughness, results in significantly smaller modeling errors than the I-model (isotropic mesoscale roughness) or the F-model (flat mesoscale surface) in areas with significant azimuth modulation. In regions where the observed azimuth modulation is larger than $1 \mathrm{~dB}$, the mean rms error is $0.46 \mathrm{~dB}$ for the A-model compared to $0.70 \mathrm{~dB}$ for the isotropic roughness models. In regions with azimuth modulation over $2 \mathrm{~dB}$, the A-model mean rms error is $0.52 \mathrm{~dB}$ compared to $0.93 \mathrm{~dB}$ for the other models.

The A-model is a promising tool for characterization of the Greenland surface. Using $\sigma^{\circ}$ azimuth modulation, the dynamics of the Greenland ice sheet may be better tracked, including long-term wind patterns. A better understanding of the azimuth modulation over Greenland in connection with geophysical surface properties may be obtained through in situ studies which combine measurements of $\sigma^{\circ}$ azimuth modulation with measurements of the geophysical properties of the surface.

\section{ACKNOWLEDGEMENTS}

ERS wind scatterometer data were kindly provided by IFREMER as part of ESA project AO2.USA.119. AWS data were kindly provided by K. Steffen and the Greenland Climate Network.

\section{REFERENCES}

Ashcraft, I.S. and D.G. Long. 2005. Observation and characterization of radar backscatter over Greenland. IEEE T. Geosci. Remote, 43(2), 237-246.

Ashcraft, I.S. and D.G. Long. In press. Comparison of methods for melt detection over Greenland using active and passive microwave measurements. IEEE T. Geosci. Remote.

Attema, E.P.W. 1991. The active microwave instrument on-board the ERS-1 satellite. Pr. Inst. Electr. Elect., 79(6), 791-799.

Attema, E.P.W. and P. Lecomte. 1998. The ERS-1 and ERS-2 wind scatterometers, system performance and data products. In Proceedings of the IEEE International Geoscience and Remote Sensing Symposium. Seattle, WA, Institute of Electrical and Electronics Engineers, 1967-1969.

Bales, R.C., J.R. McConnell, E. Mosley-Thompson and B. Csatho. 2001. Accumulation over the Greenland ice sheet from historical and recent records. J. Geophys. Res., 106(D24), 33,81333,825 .

Benson, C.S. 1962. Stratigraphic studies in the snow and firn of the Greenland ice sheet. SIPRE Res. Rep. 70.

Bromwich, D.H., T.R. Parish and C.A. Zorman. 1990. The confluence zone of the intense katabatic winds at Terra Nova Bay, Antarctica, as derived from airborne sastrugi surveys and mesoscale numerical modeling. J. Geophys. Res., 95(D5), 5495-5509.

Bromwich, D.H., Y. Du and K.M. Hines. 1996. Wintertime surface winds over the Greenland ice sheet. Mon. Weather Rev., 124(9), 1941-1947.
Broschat, S.L. and E.I. Thorsos. 1997. An investigation of the small slope approximation for scattering from rough surfaces. Part II. Numerical studies. J. Acous. Soc. Am., 101(5), 2615-2625.

Drinkwater, M.R., D.G. Long and A.W. Bingham. 2001. Greenland snow accumulation estimates from satellite radar scatterometer data. J. Geophys. Res., 106(D24), 33,935-33,950.

Fahnestock, M., R. Bindschadler, R. Kwok and K. Jezek. 1993. Greenland ice sheet surface properties and ice dynamics from ERS-1 SAR imagery. Science, 262(5139), 1530-1534.

Fristrup, B. 1966. The Greenland ice cap. Copenhagen, Rhodos, International Science Publishers.

Jezek, K.C., P. Gogineni and M. Shanableh. 1994. Radar measurements of melt zones on the Greenland ice sheet. Geophys. Res. Lett., 21(1), 33-36.

Ledroit, M., F. Rémy and J.F. Minster. 1993. Observations of the Antarctic ice sheet with the Seasat scatterometer: relation to katabatic-wind intensity and direction. J. Glaciol., 39(132), 385-396.

Long, D.G. and M.R. Drinkwater. 1994. Greenland ice-sheet surface properties observed by the Seasat-A scatterometer at enhanced resolution. J. Glaciol., 40(135), 213-230.

Long, D.G. and M.R. Drinkwater. 2000. Azimuth variation in microwave scatterometer and radiometer data over Antarctica. IEEE T. Geosci. Remote, 38(4), 1857-1870.

Marquardt, D. 1963. An algorithm for least-squares estimation of nonlinear parameters. J. Soc. Indust. Appl. Math., 11, 431-441.

Ohmura, A. and N. Reeh. 1991. New precipitation and accumulation maps for Greenland. J. Glaciol., 37(125), 140-148.

Rignot, E.J., S.J. Ostro, J.J. Zyl and K.C. Jezek. 1993. Unusual radar echoes from the Greenland ice sheet. Science, 261(5129), 1710-1713.

Rott, H. and W. Rack. 1995. Characterization of Antarctic firn by means of ERS-1 scatterometer measurements. In IGARSS'95, Quantitative Remote Sensing for Science and Applications, Proceedings, 15th International Geoscience and Remote Sensing Symposium. New York, Institute of Electrical and Electronics Engineers, 2041-2043.

Rott, H., K. Sturm and H. Miller. 1993. Active and passive microwave signatures of Antarctic firn by means of field measurements and satellite data. Ann. Glaciol., 17, 337-343.

Steffen, K., J. Box and W. Abdalati. 1996. Greenland climate network: GC-net. CRREL Spec. Rep., 96-27.

Swift, C.T. 1997. SeaSat scatterometer observations of sea ice. IEEE T. Geosci. Remote, 37(2), 716-723.

Swift, C.T., P.S. Hayes, J.S. Herd, W.L. Jones and V.E. Delnore. 1985. Airborne microwave measurements of the southern Greenland ice sheet. J. Geophys. Res., 90(B2), 1983-1994.

Ulaby, F.T., R.K. Moore and A.K. Fung. 1986a. Microwave remote sensing, active and passive. Vol. 2. Norwood, MA, Artech House, Inc.

Ulaby, F.T., R.K. Moore and A.K. Fung. 1986b. Microwave remote sensing, active and passive. Vol. 3. Reading, MA, Artech House, Inc.

Wismann, V. 2000. Monitoring of seasonal snowmelt on Greenland with ERS scatterometer data. IEEE T. Geosci. Remote, 38(4), 1821-1826.

Young, N.W., D. Hall and G. Hyland. 1996. Directional anisotropy of C-band backscatter and orientation of surface microrelief in East Antarctica. In Kingwell, J., ed. Proceedings of the First Australian ERS Symposium. Hobart, University of Tasmania, 117-126. 